\title{
Very early antiretroviral therapy permits CD8 T cells to keep HIV reservoirs at bay
}

\author{
Jean-Pierre Routy $^{1,2,3}$, Vikram Mehraj ${ }^{1,2}$ \\ ${ }^{1}$ Research Institute, ${ }^{2}$ Chronic Viral Illness Service, ${ }^{3}$ Division of Hematology, McGill University Health Centre, Montréal, Québec, Canada \\ Correspondence to: Jean-Pierre Routy, MD, FRCPC. Division of Hematology and Chronic Viral Illness Service, McGill University Health Centre: \\ Glen site, Research Institute, Block E, Suite EM 3-3232, Mezzanine 3M, 1001 Boulevard Décarie, Montréal, QC H4A 3J1, Canada. \\ Email: jean-pierre.routy@mcgill.ca. \\ Provenance: This is a Guest Editorial commissioned by Section Editor Zhijun Han, MD (Department of Laboratory Medicine, Wuxi Second \\ Hospital, Nanjing Medical University, Wuxi, China). \\ Comment on: Takata H, Buranapraditkun S, Kessing C, et al. Delayed differentiation of potent effector CD8+ T cells reducing viremia and reservoir \\ seeding in acute HIV infection. Sci Transl Med 2017;9:eaag1809.
}

Submitted Aug 18, 2017. Accepted for publication Aug 24, 2017.

doi: $10.21037 / \mathrm{atm} .2017 .08 .38$

View this article at: http://dx.doi.org/10.21037/atm.2017.08.38

Human immunodeficiency virus (HIV) infection is defined by a profound immune dysfunction and an abrogated $\mathrm{T}$ cell homeostasis (1). The gradual loss of CD4 T cells is associated with disease progression in the absence of treatment. On the other hand, CD8 T cell count becomes very elevated at the earliest phase of infection and plateaus during the chronic phase up until the very late phase, when major depletion of all $\mathrm{T}$ cell subsets occurs (2). In the majority of patients living with HIV, antiretroviral therapy (ART) suppresses HIV replication, reduces the risk for AIDS and non-AIDS events and contributes to an improved health status (3). Usually, an optimal CD4 T cell reconstitution $(>500$ cells $/ \mu \mathrm{L})$ is achieved a few years after treatment depending on CD4 $\mathrm{T}$ cell count at the time of treatment initiation (1).

\section{Temporal dynamics of CD8 $\mathbf{T}$ cells responses during HIV infection}

In contrast to the CD4 $\mathrm{T}$ cell decline, the elevation of CD8 $\mathrm{T}$ cells persists in absence of treatment and only partially decreases even after a decade of treatment. Despite the control of viral replication on ART, normalization of CD8 $\mathrm{T}$ cell counts is rarely observed even with optimal CD4 T cell reconstitution. Such persistence of elevated CD8 T cell counts independent of CD4 $\mathrm{T}$ cell reconstitution has been linked with an increased risk of non-AIDS-related clinical events, including renal diseases, cardiovascular disorders, neurocognitive dysfunction and cancer $(2,4)$. Indeed, only early initiation rather than prolonged duration of ART contributes to the normalization of CD8 $\mathrm{T}$ cell counts $(2,5)$. These observations in treated HIV infection have led to consider the CD4/CD8 ratio as a better marker than CD4 or CD8 $\mathrm{T}$ cell counts in predicting risk of inflammatory non-AIDS events, level of immune exhaustion and HIV reservoir size (6).

\section{Temporal dynamics of HIV specific CD8 T cell responses during HIV infection}

Antigen-specific CD8 T cells represent a major component of defense system against invading viruses, by recognising and killing infected cells harboring non-self-proteins. Crucial step in this process involves T cell receptor (TCR) recognition of virus-derived peptides presented on the surface of infected CD4 T cells by molecules of the human leukocyte antigen (HLA) class I.

The HIV specific CD8 T cells constitute a small fraction of the total population of CD8 $\mathrm{T}$ cells and are primed at the early phase of infection when viremia bursts. Then primed $\mathrm{HIV}$-specific CD8 $\mathrm{T}$ cell responses gain partial control over viral replication and establish a viral set point that further dictates the speed of disease progression. Even in the rare individuals who spontaneously control viral replication, 
called elite controllers, clearance is not achieved. Thus, despite their partial ability to control viremia, HIVspecific CD8 $\mathrm{T}$ cells are unable to clear HIV infection. Understanding the factors limiting CD8 T cells' ability to kill infected cells measured by their cytotoxic function has been a research priority for years. We first learned that after undergoing rapid expansion following acute HIV infection, CD8 T cells waned and became hypo-functional or "exhausted" after a few months of infection (7). The quality of CD8 T cell response contributes to the establishment of a viral set point in each patient that in turn has been shown to predict the time of the development of AIDS events and CD4 $\mathrm{T}$ cell decay $(8,9)$. Furthermore, the contribution of CD8 $\mathrm{T}$ cell function was illustrated by the ability of a fraction of elite controllers carrying distinctive HLA-B57 or HLA-B27 alleles to suppress viral replication (10); and by viral escape due to the immune pressure observed after early infection. It is now established that such emergence of HIV specific response have been temporally associated with plasma viral load decay following the peak of viremia during the acute infection. The temporal dynamics of HIV specific CD8 T cell cytotoxic response to become exhausted remains poorly defined (11).

\section{Influence of early ART initiation on the dynamics of HIV specific CD8 T cell responses}

Conversely to other viral specific responses (for example infection with cytomegalovirus), HIV-specific CD8 T cell responses following primary infection, rapidly wane following ART initiation and are linked with an irreversible cell surface expression of an immune checkpoint receptor called programmed cell death protein 1 (PD-1) leading to a persistent cellular memory defect $(7,12)$.

To gain further insight on the dynamics of CD8 T cell function on the control of viremia under ART, Cartwright and Silvestri et al. conducted a very elegant study showing that antibodies blocking CD8 $\mathrm{T}$ cells led to a viral rebound in viremic simian immunodeficiency virus (SIV)-infected macaques a few months after ART initiation at the time of infection (13). They further showed that repopulation of CD8 T cells was associated with prompt reestablishment of viral control. These study findings highlighted the role of SIV-specific CD8 $\mathrm{T}$ cells on both viral production in blood and SIV reservoir size measured in tissues (14). Such animal model experiments demonstrated the role of CD8 T cell function on the control of pre ART viremia and size of viral reservoir (total DNA load) following early ART.
Can a similar observation from SIV-infected macaque be observed in very early treated HIV-infected patients? Our limited understanding of the contribution of CD8 $\mathrm{T}$ cell functional dynamics in early HIV infection is due to the inability to recruit and treat patients within the first week of infection as the mean time to participation in acute HIV infection research cohorts is around one to three months after infection $(5,11,15)$. Such limitation was recently overcome by establishing a unique cohort (RV254/SEARCH010) in Thailand (16). This cohort was implemented after frequent screening of thousands of individuals in HIV voluntary counselling and testing centers who were invited to be treated within days of acquiring HIV infection.

Trautmann et al. who previously reported on progressive exhaustion of cytolytic function of HIV-specific CD8 T cells, after two to four months of HIV infection (17), now assessed such response within days of infection in participants of the Thai cohort. These participants received treatment during very early HIV infection and were grouped as stage 1, 2 and 3 with a median of 14,16 and 19 days post-infection, respectively (18). They were able to show that the magnitude of HIV-specific CD8 T cells and their proliferative capacity were delayed on ART during stages 1 and 2 occurring before the peak of viremia. Despite very early appearance of HIV-specific CD8 T cells, their expansion and function were abrogated with treatment. These results are in line with previous findings in SIV model where similar low CD8 T cell responses were observed in mucosal tissues following early infection (19). Conversely, at the time of the peak viremia (stage 3, median of 23 days post HIV-infection) a massive expansion of HIVspecific CD8 T cells occur concomitant to the burst of cytokine response and symptoms of acute infection (20). The fully differentiated HIV specific CD8 T cells led to a shorter time to control viral replication compared to groups 1 and 2. Most importantly, an elevated capacity to maintain HIV specific CD8 T cell function was linked to a lower viral reservoir size measured by HIV DNA PCR. Globally, these study findings showed that HIV specific CD8 T cell response contribute to the reduction of the pool of HIV producing cells as well as the HIV reservoir size in early ART treated patients.

Such study findings, which represent a tour de force, pave the way for the development of strategy that could enhance $\mathrm{HIV}$-specific functions. However, the generalizability of this study is limited as the median age of the participants was 27 years, most were male (89\%), and $76 \%$ harboured a clade 
CRF01_AE HIV which is known to have a faster disease progression than clade B (21). Furthermore, this study was confined to the analysis of blood samples, opening avenues to analyze lymph node and gut associated lymphoid tissues where the majority of HIV reservoir resides. However, access to the human tissue samples remains an issue in HIV clinical research and needs to balance the risks/benefits to the participants (22). Lastly, this type of comprehensive research infrastructure like the one in Thailand allowing frequent screening for HIV acquisition combined with a very early treatment is not scalable.

\section{Future directions}

As the peak of viremia predicts viral set point, indicating that very early events are at play before CD8 $\mathrm{T}$ cells kick in (23). Innate immune response that occurs within hours induces production of type I interferons (IFNs), which regulate many immune pathways including the function of CD8 T cells. It is well established that in acute lymphocytic choriomeningitis virus (LMCV) infection mice model, IFNs helps to clear infection, while in chronic HIV infection higher levels of type I IFN signaling is correlated with immune activation, disease progression and reduced CD4 T cell recovery on ART. More information on the "IFN paradox" and CD8 T cell response in early infection is warranted (24).

Barouch et al. in a landmark study analyzed the earliest events following mucosal SIV infection of rhesus monkeys (25). They evaluated SIV CD8 T cell responses in blood and in several tissues on day $0,1,3,7$ and 10 postinfection. The earliest SIV specific CD8 $\mathrm{T}$ cell responses were observed on day 7 in female reproductive tract, gastrointestinal mucosa and bone marrow. Such early responses were not observed in lymph nodes and blood. Subsequently all monkeys exhibited high-frequency of specific CD8 $\mathrm{T}$ cell responses on day 10 in all tissues except blood.

These results demonstrate that during acute infection SIV specific responses first develop in tissue effector sites prior to the emergence of responses into the periphery, thus occurring "too little and too late" to block the viral swarm (19). They further assessed the transcriptomic signatures and observed direct strong correlation of SIVspecific CD8 $\mathrm{T}$ cell responses with metabolic pathways in tissues where such pathways are involved in $\mathrm{T}$ cell activation. They further observed an inverse correlation of immunosuppressive cytokine transforming growth factor beta 1 (TGF- $\beta 1$ ) response genes with the magnitude of CD8 $\mathrm{T}$ cell responses on days 7 and 10 in gastrointestinal tissues demonstrating the inhibition of effective CD8 T cell responses at the very sites of early virus replication. These results further indicate that effective HIV-specific CD8 $\mathrm{T}$ cells play an important role in the reduction of HIVproducing cells and more importantly latent reservoirs in patients receiving early ART. In these lines, use of latency reversing agents in vitro such as vorinostat has been shown to create a window of vulnerability by inducing production of viral protein on the cell surface allowing for subsequent clearance of latently infected cells by an array of effector mechanisms including ex vivo autologous CD8 T cells (26).

Globally, all these findings in SIV and HIV infections established a pathogenesis link between CD8 T cell function and both viral load and size of HIV reservoir on ART. However, only comprehensive integrated strategies that will encompass enhancing innate cells, IFNs, antibodies and specific CD8 T cell responses with adequate CD4 T cell help (27) will provide long-term viral control or an HIV cure.

\section{Acknowledgements}

The authors are grateful to Angie Massicotte for administrative assistance and Nikola Kokinov for critical reading of the manuscript.

Funding: This work was supported by the Fonds de Recherche du Québec - Santé (FRQ-S): Réseau SIDA/ Maladies infectieuses and ThéCell; the Canadian Institutes of Health Research (CIHR; grant MOP 103230 and IBC 154051); the Vaccines \& Immunotherapies Core of the CIHR Canadian HIV Trials Network (CTN; grant CTN 257); the Canadian Foundation for AIDS Research (CANFAR; grant 02-512); and the Canadian HIV Cure Enterprise Team Grant (HIG-133050) awarded by the CIHR in partnership with CANFAR. JP Routy is the holder of the Louis Lowenstein Chair in Hematology and Oncology, McGill University. V Mehraj is supported by an FRQ-S Postdoctoral Fellowship Award.

\section{Footnote}

Conflicts of Interest: The authors have no conflicts of interest to declare.

\section{References}

1. Maartens G, Celum C, Lewin SR. HIV infection: 
epidemiology, pathogenesis, treatment, and prevention. Lancet 2014;384:258-71.

2. Cao W, Mehraj V, Kaufmann DE, et al. Elevation and persistence of CD8 T-cells in HIV infection: the Achilles heel in the ART era. J Int AIDS Soc 2016;19:20697.

3. Ford N, Meintjes G, Vitoria M, et al. The evolving role of CD4 cell counts in HIV care. Curr Opin HIV AIDS 2017;12:123-8.

4. Sinha A, Ma Y, Scherzer R, et al. Role of T-Cell Dysfunction, Inflammation, and Coagulation in Microvascular Disease in HIV. J Am Heart Assoc 2016;5:e004243.

5. Cao W, Mehraj V, Trottier B, et al. Early Initiation Rather Than Prolonged Duration of Antiretroviral Therapy in HIV Infection Contributes to the Normalization of CD8 T-Cell Counts. Clin Infect Dis 2016;62:250-7.

6. Lu W, Mehraj V, Vyboh K, et al. CD4:CD8 ratio as a frontier marker for clinical outcome, immune dysfunction and viral reservoir size in virologically suppressed HIVpositive patients. J Int AIDS Soc 2015;18:20052.

7. Trautmann L, Janbazian L, Chomont N, et al. Upregulation of PD-1 expression on HIV-specific CD8+ $\mathrm{T}$ cells leads to reversible immune dysfunction. Nat Med 2006;12:1198-202.

8. Streeck H, Jolin JS, Qi Y, et al. Human immunodeficiency virus type 1-specific CD8+ T-cell responses during primary infection are major determinants of the viral set point and loss of CD4+ T cells. J Virol 2009;83:7641-8.

9. Streeck H, Lu R, Beckwith N, et al. Emergence of individual HIV-specific CD8 $\mathrm{T}$ cell responses during primary HIV-1 infection can determine long-term disease outcome. J Virol 2014;88:12793-801.

10. Altfeld M, Kalife ET, Qi Y, et al. HLA Alleles Associated with Delayed Progression to AIDS Contribute Strongly to the Initial CD8(+) T Cell Response against HIV-1. PLoS Med 2006;3:e403.

11. Demers KR, Makedonas G, Buggert M, et al. Temporal Dynamics of CD8+ T Cell Effector Responses during Primary HIV Infection. PLoS Pathog 2016;12:e1005805.

12. Gray CM, Lawrence J, Schapiro JM, et al. Frequency of class I HLA-restricted anti-HIV CD8+ T cells in individuals receiving highly active antiretroviral therapy (HAART). J Immunol 1999;162:1780-8.

13. Cartwright EK, Spicer L, Smith SA, et al. CD8(+) Lymphocytes Are Required for Maintaining Viral Suppression in SIV-Infected Macaques Treated with ShortTerm Antiretroviral Therapy. Immunity 2016;45:656-68.
14. Roberts ER, Carnathan DG, Li H, et al. Collapse of Cytolytic Potential in SIV-Specific CD8+ T Cells Following Acute SIV Infection in Rhesus Macaques. PLoS Pathog 2016;12:e1006135.

15. Mehraj V, Cox J, Lebouché B, et al. Socioeconomic status and time trends associated with early ART initiation following primary HIV infection in Montreal, Canada: 1996-2015. 9th IAS Conference on HIV Science (IAS 2017); Paris, 2017.

16. Ananworanich J, Schuetz A, Vandergeeten C, et al. Impact of multi-targeted antiretroviral treatment on gut $\mathrm{T}$ cell depletion and HIV reservoir seeding during acute HIV infection. PLoS One 2012;7:e33948.

17. Trautmann L, Mbitikon-Kobo FM, Goulet JP, et al. Profound metabolic, functional, and cytolytic differences characterize HIV-specific CD8 T cells in primary and chronic HIV infection. Blood 2012;120:3466-77.

18. Takata H, Buranapraditkun S, Kessing C, et al. Delayed differentiation of potent effector CD8+ T cells reducing viremia and reservoir seeding in acute HIV infection. Sci Transl Med 2017;9:eaag1809.

19. Reynolds MR, Rakasz E, Skinner PJ, et al. CD8+ T-lymphocyte response to major immunodominant epitopes after vaginal exposure to simian immunodeficiency virus: too late and too little. J Virol 2005;79:9228-35.

20. Stacey AR, Norris PJ, Qin L, et al. Induction of a striking systemic cytokine cascade prior to peak viremia in acute human immunodeficiency virus type 1 infection, in contrast to more modest and delayed responses in acute hepatitis $\mathrm{B}$ and $\mathrm{C}$ virus infections. J Virol 2009;83:3719-33.

21. Li Y, Han Y, Xie J, et al. CRF01_AE subtype is associated with $\mathrm{X} 4$ tropism and fast HIV progression in Chinese patients infected through sexual transmission. AIDS 2014;28:521-30.

22. Mehraj V, Ghali MP, Ramendra R, et al. The evaluation of risk-benefits ratio for gut tissue sampling in HIV cure research. J Virus Erad 2017;3:212-7.

23. Robb ML, Eller LA, Kibuuka H, et al. Prospective Study of Acute HIV-1 Infection in Adults in East Africa and Thailand. N Engl J Med 2016;374:2120-30.

24. Deeks SG, Odorizzi PM, Sekaly RP. The interferon paradox: can inhibiting an antiviral mechanism advance an HIV cure? J Clin Invest 2017;127:103-5.

25. Barouch DH, Ghneim K, Bosche WJ, et al. Rapid Inflammasome Activation following Mucosal SIV Infection of Rhesus Monkeys. Cell 2016;165:656-67. 
26. Sung JA, Sholtis K, Kirchherr J, et al. Vorinostat Renders the Replication-Competent Latent Reservoir of Human Immunodeficiency Virus (HIV) Vulnerable to Clearance by CD8 T Cells. EBioMedicine 2017;23:52-8.

Cite this article as: Routy JP, Mehraj V. Very early antiretroviral therapy permits CD8 T cells to keep HIV reservoirs at bay. Ann Transl Med 2017;5(21):434. doi: 10.21037/ atm.2017.08.38
27. Deeks SG, Lewin SR, Ross AL, et al. International AIDS Society global scientific strategy: towards an HIV cure 2016. Nat Med 2016;22:839-50. 